\title{
Fully Integrated Applications of Thin Films on Low Temperature Cofired Ceramic (LTCC)
}

\author{
Ambrose Wolf ${ }^{1}$, Ken Peterson ${ }^{2}$, Matt O'Keefe ${ }^{3}$, Wayne Huebner ${ }^{3}$, Bill Kuhn ${ }^{4}$ \\ ${ }^{1}$ NNSA's Kansas City Plant, Kansas City, MO USA \\ *Phone: 816-997-2127 Email: awolf@kcp.com \\ ${ }^{2}$ Sandia National Laboratories, Albuquerque, NM, USA, \\ ${ }^{3}$ Missouri University of Science and Technology (MS\&T) \\ ${ }^{4}$ Kansas State University (K-State)
}

\begin{abstract}
Thin film multilayers have previously been introduced on multilayer low temperature cofired ceramic (LTCC), as well as initial thin film capacitors on LTCC. The ruggedness of a multipurpose Ti-Cu-Pt-Au stack for connectivity and RF conductivity has continued to benefit fabrication and reliability in state of-the-art modules, while the capacitors have followed the traditional Metal-Insulator-Metal (MIM) style. The full integration of thin film passives with thin film connectivity traces is presented. Certain passives, such as capacitors, require specifically tailored and separately patterned thin film (multi-)layers, including a dielectric. Different capacitance values are achieved by variation of both the insulator layer thickness and the active area of the capacitor. Other passives, such as filters, require only the conductor - a single thin film multilayer. This can be patterned from the same connectivity thin film material (Ti-Cu-Pt-Au), or a specially tailored thin film material (e.g. Ti-Cu-Au) can be deposited. Both versions are described, including process and integration details. Examples are discussed, ranging from patterning for maximum tolerances, to space and performance-optimized designs. Cross-sectional issues associated with integration are also highlighted in the discussion.
\end{abstract}

The Kansas City Plant is operated by Honeywell Federal Manufacturing \& Technologies for the United States Department of Energy under Contract No. DE-NA0000622. Sandia is a multiprogram laboratory operated by Sandia Corporation, a Lockheed Mar04-94AL85000.

Key Words: LTCC, thin film, PVD

Low Temperature Co-fired Ceramic (LTCC) is a technology that has been readily adapted to many packaging applications, the varieties of which are expanding. In the RF area LTCC has been used for Multi-Chip Modules (MCM) involving additional capacitors and filters. Printed wiring boards are attractive due to the low cost and low dielectric constants for signal propagation, whereas LTCC has frequently been selected where material stability over special (harsh) environments is required. Soldering to LTCC involves a reaction with the thick film which is the adhesion layer - limiting the ruggedness and reworkability. Recently thin film materials on LTCC have been developed to improve not only the solder processing, but also the wire bond connectivity, good RF conductivity and highly increased ruggedness. Further thin film advantages are edges with nearvertical side walls (ion milling used for pattern creation) and bulk material properties. Methods to handle drawbacks (e.g. rough surface topography on vias mitigated by thick film cover pads) have been developed. This manuscript reports on the first full integration of thin film capacitors (Fig. 1) and filters on an LTCC MCM.
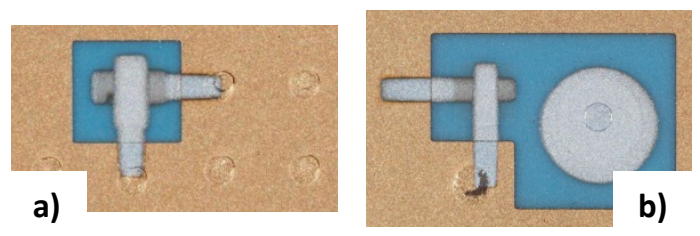

c)
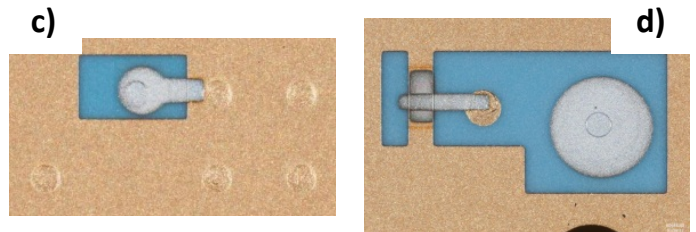

Figure 1: Thin film MIM capacitors integrated with $\mathrm{Ti} / \mathrm{Cu} / \mathrm{Pt} / \mathrm{Au}$ connectivity thin film prior to ion milling for trace pattern creation. Parts a) and b) are designs for high tolerance, c) and d) the same locations with optimized design (cf. Fig. 2).

Full integration of thin film capacitors on LTCC is consistent with higher reliability and ruggedness of the entire MCMC system. Initial standalone test capacitors were designed to develop the PVD material deposition on LTCC as well as the electrode/dielectric synergy necessary for functional capacitors. Sputter deposition of the two electrodes and the dielectric layer were performed using 
physical masks for device definition. The prototype multilayer that attained the desired $1-50 \mathrm{pF}$ capacitance range was determined to be $200 \mathrm{~nm} \mathrm{Al} /$ $1,000 \mathrm{~nm} \mathrm{Al} 2 \mathrm{O} 3 / 200 \mathrm{~nm}$ Al. Fine-tuning to the exact desired capacitance value is then achieved via active capacitor area, ranging on the order of $125 \mu \mathrm{m}^{2}$ to $1,000 \mu \mathrm{m}^{2}$. Testing performed up to the single digit $\mathrm{GHz}$ range demonstrated good temperature and frequency stability of less than $6.5 \%$ and a low loss with an equivalent series resistance (ESR) of approximately $0.5 \Omega$, aligning the devices with $\mathrm{F}$ type Electronic Industries Alliance (EIA) class II capacitors. This prototype recipe was used to make the first fully integrated devices on an MCM module (Fig. 1 and 2).

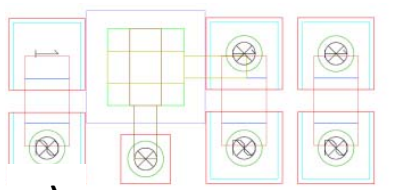

a)

c)

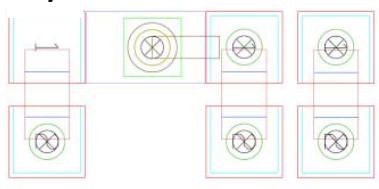

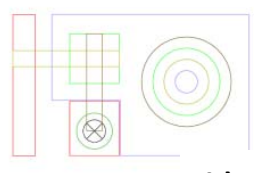

b)

d)

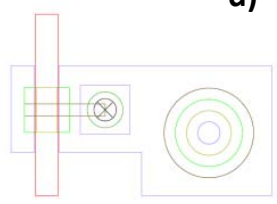

Figure 2. AutoCAD design layout of for MIM capacitors in Fig. 1, with some traces, surface vias, soldered COTS parts and mask alignment targets visible. Parts a), b), c) and d) same as Fig. 1.

After coating the entire LTCC panel with $\mathrm{Ti} / \mathrm{Cu} / \mathrm{Pt} / \mathrm{Au}$ to create the basis for the circuit, windows were ion-milled at the thin film capacitor locations. This was done both on plain LTCC as well as over a connecting filled via. After this, physical masks were used in the PVD deposition of the threelayer MIM capacitor structure, overlapping the electrodes onto the appropriate location of the remaining connectivity thin film for signal integration (Fig. 1). Two designs for the capacitor were used, one for maximum tolerance such that misalignment of the physical masks would not cause device shorting (Fig. 2 a) and b)). This was achieved by using a 90 degree overlap of the two electrodes with extended electrode length. The other design optimizes the electrode structure for shortened leads (Fig. 2 c) and d)). In both of these cases the electrical function is to conduct the signal through the capacitor to ground, which is achieved by locating the capacitor directly on top of the ground via and thus eliminating the lead length. The remaining step is to ion-mill the main thin film circuit, while protecting the thin film capacitor devices, as indicated in Fig. 2.

The full integration of filters was approached in a slightly different way. The selected test filter requires only a single multilayer conductor, in this case $50 \mathrm{~nm} \mathrm{Ti} / 6,000 \mathrm{~nm} \mathrm{Cu} / 375 \mathrm{~nm} \mathrm{Au}$. The entire panel was coated with $\mathrm{Ti} / \mathrm{Cu} / \mathrm{Pt} / \mathrm{Au}$ in a similar fashion. A window was ion-milled to prepare for the thin film filter material, leaving a connecting finger for the thin film transition. A second PVD process was performed using a physical mask, filling only the ion-milled window with the $\mathrm{Ti} / \mathrm{Cu} / \mathrm{Au}$ multilayer, overlapping the connective finger while staying away from the remaining thin film edge. After this the entire pattern, i.e. traces, solder and wire bond pads as well as filter geometry, is ion-milled. Fig. 3 shows the transition between the $\mathrm{Ti} / \mathrm{Cu} / \mathrm{Pt} / \mathrm{Au}$ (left edge of pictures) and the device $\mathrm{Ti} / \mathrm{Cu} / \mathrm{Au}$ layer (right edge of pictures).

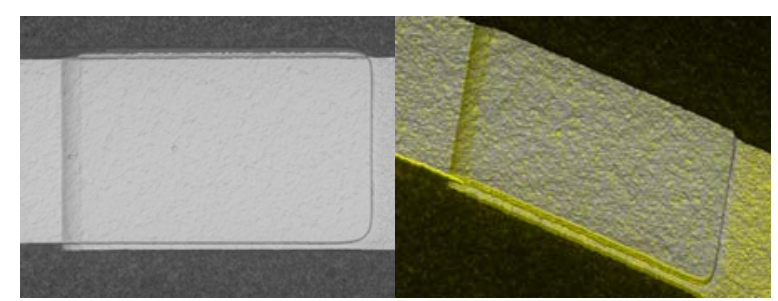

Figure 3. SEM of Thin Film transition between standard multipurpose $\mathrm{Ti} / \mathrm{Cu} / \mathrm{Pt} / \mathrm{Au}$ for connectivity (left edge) and special $\mathrm{Ti} / \mathrm{Cu} / \mathrm{Au}$ for device functionality (right edge).

A closer examination of Fig. 3 demonstrates the difference between the edge structures, where the edge on the left side was created with a physical mask and shows the typical sloped step. The remaining edges show the near-vertical definition achieved by ion-milling. It is noteworthy that the thin film multilayer on the right side if each picture overlaps on top of the thin film multilayer on the left side. This fact is not obvious from a cursory. The exact transition across the near-vertical ion-milled edge still needs to be revealed by cross sectioning (in work). The electrical testing of the filter functionality is ongoing.

In conclusion, the full integration of thin film capacitors and filters has been achieved. This work opens the door to a much broader range of devices including, among others, high-k dielectric materials (e.g., Barium Titanate) and other passives such as resistors and inductors.

Notice: This manuscript has been authored by Honeywell Federal Manufacturing \& Technologies under Contract No.DE-NA-0000622 with the U.S. Department of Energy. The United States Government retains and the publisher, by accepting the article for publication, acknowledges that the United States Government retains a 
nonexclusive, paid-up, irrevocable, world-wide license to publish or reproduce the published form of this manuscript, or allow others to do so, for United States Government purposes. 